\title{
Rheumatoid arthritis in patients with HIV: management challenges
}

This article was published in the following Dove Press journal:

Open Access Rheumatology: Research and Reviews

29 April 2016

Number of times this article has been viewed

\section{Matthew B Carroll \\ Joshua H Fields \\ Philip G Clerc}

Department of Rheumatology, Keesler Medical Center, Keesler Air Force Base, Biloxi, MS, USA
Correspondence: Matthew B Carroll Keesler Medical Center, 30I Fisher Avenue, Keesler Air Force Base, Biloxi, MS 39534, USA

Tel +I 2283763629

Fax + I 2283760184

Email matthew.carroll.I@us.af.mil
Abstract: Over the past few decades, HIV has been transformed from a once-uniformly fatal disease to now a manageable but complex multisystem illness. Before highly active antiretroviral therapy (HAART), reports suggested that HIV-infected patients with rheumatoid arthritis (RA) would experience remission of their disease. It has now become clear that RA can develop in HIV-infected patients at any time, independent of HAART. Choosing the right medication to treat symptoms related to RA while avoiding excess weakening of the immune system remains a clinical challenge. Agents such as hydroxychloroquine and sulfasalazine might best balance safety with efficacy, making them reasonable first choices for therapy in HIV-infected patients with RA. More immune suppressing agents such as methotrexate may balance safety with efficacy, but data are limited. Corticosteroids such as prednisone may also be reasonable but could increase the risk of osteonecrosis. Among biologic response modifiers, tumor necrosis factor $\alpha$ inhibitors may balance safety with efficacy, but perhaps when HIV replication is controlled with HAART. Monitoring RA disease activity remains challenging as only one retrospective study has been published in this area. Those with HIV infection and RA can experience comorbidities such as accelerated heart disease and osteoporosis, a consequence of the chronic inflammatory state that each illness generates. Although HIV-infected patients are at risk for developing the immune reconstitution inflammatory syndrome when starting HAART, it appears that immune reconstitution inflammatory syndrome has a minimal effect on triggering the onset or the worsening of RA.

Keywords: human immunodeficiency virus, rheumatoid arthritis, osteoporosis, cardiovascular disease, immune reconstitution inflammatory syndrome

\section{Introduction}

Since the early days of the HIV epidemic, various musculoskeletal and rheumatic manifestations have been recognized in HIV-infected patients. Different types of arthralgias and arthritis including, but not limited to the painful articular syndrome, HIV-associated arthritis, the seronegative spondyloarthropathies, vasculitis, and diffuse infiltrative lymphocytosis syndrome, have been reported. ${ }^{1}$ More than $5 \%$ of patients with HIV infection may experience arthralgias or arthritis, with some publications suggesting a prevalence of up to $12 \%$ for HIV-arthritis. ${ }^{2,3}$ Contrary to the relationship between these arthritis syndromes and HIV infection, reports early on the HIV epidemic suggested that those with HIV infection who also had rheumatoid arthritis (RA) entered remission. ${ }^{4}$ Over the past 2 decades, HIV infection has been transformed from an untreatable and uniformly fatal illness to a manageable chronic illness. ${ }^{5}$ Multiple publications now support the premise that RA can coexist with 
HIV infection and can develop before or after HIV infection occurs, even independent of the initiation of highly active antiretroviral therapy (HAART), although the coexistence of the two illnesses is infrequent. ${ }^{3,6,7}$ Numerous challenges confront providers who treat HIV-infected patients presenting with a symmetry large and small joint polyarthritis suspicious for RA. Chronic HIV infection predisposes patients to an increased risk of systemic autoimmunity in general. ${ }^{8,9}$ One study demonstrated that anticyclic citrullinated peptide antibodies can be detected at a low-to-moderate level in up to $15 \%$ of HIV-infected patients with advanced disease, with levels decreasing after the initiation of HAART. ${ }^{10}$ It has also been reported that HIV-infected patients without any rheumatic complaints had a median value of both anticyclic citrullinated peptide antibodies and rheumatoid factor significantly higher than controls. ${ }^{8,11}$ When a diagnosis of RA is made in an HIV-infected patient, the treating clinician faces numerous challenges that range from choosing an appropriate medication that balances safety with efficacy to mitigating the effects of chronic inflammation. ${ }^{3}$ The purpose of this review was to explore in depth the management of RA in patients with HIV infection. We critically examine the currently available medical literature, focusing on the role of disease-modifying antirheumatic drugs (DMARDs) and biologic response modifiers in HIV-infected patients with RA. We also review how to measure RA disease activity, monitor for comorbidities that may develop as a consequence of chronic infection with HIV and inflammation from RA and investigate the effect of the immune reconstitution inflammatory syndrome (IRIS) either unmasking RA or triggering its exacerbation.

\section{Pharmacologic options for treating rheumatoid arthritis in HIV infection Disease-modifying antirheumatic drugs}

DMARDs typically used in the treatment of RA include medications, such as hydroxychloroquine (HCQ), sulfasalazine (SSZ), methotrexate (MTX), leflunomide (LFN), cyclosporine $\mathrm{A}(\mathrm{Cs} \mathrm{A})$, and corticosteroids. As a group, the majority of these medications have been available for several decades with well-defined safety profiles. The degree of immunosuppression varies with each of these medications, with HCQ and SSZ typically considered less immunosuppressive when compared to MTX or LFN. The American College of Rheumatology (ACR) "2008 Recommendations for the Use of Nonbiologic and Biologic Disease-Modifying Antirheumatic Drugs in Rheumatoid Arthritis" and the 2012 update do not make formal recommendations about which, if any, DMARD balances safety with efficacy when treating
HIV-infected patients with RA. ${ }^{12,13}$ During our review of the literature, we found that most practitioners treating HIVinfected patients with RA initiated therapy with one or more DMARDs, typically HCQ or SSZ first, before advancing therapy. The next few paragraphs summarize the findings of our review.

HCQ and its analog chloroquine have immunomodulatory and anti-inflammatory properties that make them useful in the treatment of many inflammatory diseases. HCQ has been reported to have anti-HIV properties in vitro and in vivo, resulting from its ability to increase endosomal $\mathrm{pH}$ that inhibits posttranslational modification of gp 120. ${ }^{14-17}$ While reported to have achieved remission of inflammatory arthritis in at least one patient with the acquired immunodeficiency syndrome (AIDS), ${ }^{18} \mathrm{HCQ}$ has paradoxically been reported in several clinical trials to accelerate the decline in cluster of differentiation (CD)4 T-cell counts in those not on antiretroviral therapy. ${ }^{19,20} \mathrm{~A}$ randomized control trial demonstrated an accelerated decline in CD4 T-cell counts and a significant increase in viral load in those not on HAART who received HCQ as compared to placebo. ${ }^{19}$ In contrast, another study of HIV patients who were on HAART but were nonresponders (suboptimal CD4 T-cell increases on therapy) showed that HCQ significantly increased the percentage of CD4 T-cells, which was retained 2 months after HCQ was stopped. ${ }^{21}$ There have been mixed findings on the effects of HCQ on proinflammatory cytokines and CD8 T-cell activation. ${ }^{15,19,22,23}$ One of these studies reported an excess of influenza-like illnesses in those treated with HCQ as compared to controls, but there was no increased incidence of HCQ-related retinopathy. ${ }^{19}$ Reversible hearing loss was described in one case of an HIV-infected patient who was treated with HCQ for his RA, ${ }^{24}$ but this side effect has also been reported in those without HIV infection. ${ }^{25}$ Together these results suggest that HCQ may be safe and well tolerated in patients with HIV taking HAART but also has the potential to accelerate the immunosuppressive effect of HIV, especially in those not taking HAART.

SSZ exerts immunomodulatory effects on T-cells that could translate into reduced HIV infectivity through inhibition of nuclear factor- $\kappa \mathrm{B}$ migration to the nucleolus. In vitro SSZ was shown to significantly reduce HIV infectivity and p24 antigen production. ${ }^{26}$ When combined with the protease inhibitor (PI) indinavir, the reduction was even greater than with SSZ or indinavir alone. ${ }^{26}$ Siva and Brasington ${ }^{27}$ described a patient with HIV infection who had RA and was treated with SSZ. The patient tolerated this DMARD, although his clinical course was complicated by worsening RA once 
HAART was started. ${ }^{27}$ Beyond possible use in RA, a small cohort of 14 Zambian patients treated with SSZ for HIVrelated spondyloarthropathy experienced a twofold reduction in subjective pain, joint tenderness, and joint swelling and improved clinical assessment scores after 6 months of treatment. ${ }^{28}$ Only two of these patients did not respond to SSZ therapy, and the remainder had a varied response, with most noting near-maximal improvement after 3 months. Importantly, no case of hepatic, renal, or hematologic toxicity was observed in these patients. In a small cohort of three HIV-positive patients with reactive arthritis, SSZ therapy was shown to significantly improve CD4 T-cell counts by a mean of 227 cells $/ \mathrm{mm}^{3}{ }^{29}$

Little has been reported on the use of MTX in HIVinfected patients with RA, although clinical trials are currently underway. ${ }^{30,31}$ Beyond use in RA, two HIV-positive patients with psoriasis who were treated with low-dose MTX did not develop opportunistic infections (OIs) during treatment. ${ }^{32}$ Although there have been no reports on the efficacy of LFN in HIV-infected patients with RA, one double-blind randomized trial of LFN in patients with HIV but not receiving HAART showed stable CD4 T-cell counts after 28 days of LFN therapy. ${ }^{33}$ Furthermore, LFN decreased the cycling and activation of CD4 T-cells as well as the expression of HIV coreceptors $\mathrm{C}-\mathrm{C}$ chemokine receptor type 5 and $\mathrm{C}-\mathrm{X}-\mathrm{C}$ chemokine receptor type $4 .{ }^{33,34}$ Although these serologic findings were favorable, the duration of treatment and LFN serum levels in these studies were much shorter than typically used in clinical practice, thus longer-term studies are needed to confirm sustained safety and support efficacy.

The intracellular enzyme cyclophilin A has been shown to enhance HIV viral activity, interacting with the HIV-1 gag protein. ${ }^{35}$ Consequently, cyclophilin A inhibitors, such as CsA, have been shown to inhibit HIV replication in vitro by interfering with reverse transcription and cyclophilin $\mathrm{A}$ incorporation into virions. ${ }^{36,37}$ In vivo, CsA has demonstrated some potential benefit in patients with HIV infection. One study demonstrated stable CD4 T-cell counts in HIV-infected patients after a median treatment time of 11 months on CsA, while CD4 T-cell counts decreased at a rate of 50 cells/ $\mathrm{mm}^{3}$ per year upon $\mathrm{CsA}$ cessation. ${ }^{38} \mathrm{~A}$ different randomized double-blind study had a subtly different outcome, noting that "low-dose" CsA used in early HIV infection did not suppress immune activation or increase CD4 T-cell counts. ${ }^{39}$ In fact, CsA in this study led to a small but statistically significant rise in HIV RNA levels. ${ }^{39}$ None of these studies reported an increased incidence of CsA toxicity compared to the nonHIV population.
Studies published over the past 2 to 3 decades have demonstrated that corticosteroids do not enhance HIV progression or CD4 T-cell decline in those not receiving HAART. ${ }^{40}$ In one randomized placebo-controlled trial, 8 weeks of prednisone therapy in patients with advanced HIV infection (median CD4 T-cell count of 131 cells $/ \mathrm{mm}^{3}$ ) did not significantly change CD4 T-cell counts or HIV RNA levels. ${ }^{41}$ A different study conducted among patients with lessadvanced HIV (median CD4 T-cell count of 387 cells $/ \mathrm{mm}^{3}$ ) who were receiving HAART found that prednisone had increased CD4 T-cell counts by a median of 172 cells $/ \mathrm{mm}^{3}$ after 8 weeks of treatment. ${ }^{42}$ On the contrary, another study looking at the effects of prednisolone found the opposite, specifically a trend toward lower CD4 T-cell counts in those receiving HAART compared to those who were therapy naive. ${ }^{43}$ Overall, there is evidence to suggest that corticosteroids are efficacious and reasonably safe in treating an inflammatory arthritis without accelerating the course of HIV infection, but since both HIV infection and corticosteroids can independently increase the risk of osteonecrosis, it would be prudent to limit long-term use. ${ }^{42,44,45}$

Finally, when starting a DMARD in a patient with HIV, special attention should be paid to drug interactions with HAART. Perhaps the most significant and commonly encountered interactions occur with PI given their inhibitory effect on the cytochrome P450 3A4 enzyme. Ritonavir, a PI commonly used to "boost" other PIs, is a particularly potent inhibitor of cytochrome P450 3A4. As such, PIs can increase the serum concentration of MTX, CsA, and corticosteroids, potentially to toxic levels. ${ }^{46}$ Glucocorticoids are especially vulnerable to pharmacokinetic fluctuations in the presence of PIs. A case report described a patient who developed cushingoid features after a single intra-articular injection of triamcinolone. ${ }^{47}$ It has also been reported that side effects related to corticosteroids in patients on a PI were mitigated when the corticosteroid dose was reduced by $80 \% .{ }^{48}$ Fewer and less severe drug interactions have been reported with HAART therapy involving only nucleoside and non-nucleoside reverse transcriptase inhibitors. ${ }^{49}$ More frequent laboratory monitoring should be considered when tenofovir/efavirenz/emtricitabine is prescribed with most DMARDs as this regimen has been associated with hepatic or renal toxicity, independent of that which could occur with some DMARDs.

\section{Biologic response modifiers}

The biologic response modifiers (or biologic agents) are a diverse and ever expanding group of pharmacologic agents 
that target specific cytokine or cell receptors to exert an immunosuppressive and anti-inflammatory effect. Biologic agents currently include medications that inhibit tumor necrosis factor (TNF)- $\alpha$ inhibitors, the anti-CD20 antibody rituximab, the inhibitor of T-cell costimulation abatacept, the interleukin-6 (IL-6) receptor antibody tocilizumab, and more recently the janus kinase (JAK) inhibitor tofacitinib. In general, practitioners may be hesitant to use biologic agents in patients with HIV infection and RA due to the increased risks of both infection and autoimmunity (especially with TNF- $\alpha$ inhibitors) in patients already at increased risk for either of these comorbidities. Formal guidelines regarding how to safely utilize biologic agents in HIV-infected patients with RA are limited. In their 2008 guidelines, the ACR stated, "although the panel considered contraindications for RA therapy in the setting of HIV, no recommendations concerning contraindications were issued". ${ }^{12}$ The 2012 update of the 2008 guidelines provided no new recommendations about the use of biologic agents in HIV-infected patients with RA. ${ }^{13}$ New general guidelines are anticipated in mid-2015. ${ }^{50}$ Subsequently, we summarize the available literature regarding the use and outcomes of various biologic agents used to treat RA in HIV-infected patients.

At present, the most information about available biologic agents pertains to the use of TNF- $\alpha$ inhibitors in the treatment of HIV-infected patients with RA. In 2007, Kaur et $\mathrm{al}^{51}$ reported the use of a TNF- $\alpha$ inhibitor to treat RA in an individual with HIV infection. The patient had active synovitis and failed less immunosuppressive treatment with HCQ, intraarticular steroid injections, SSZ, and oral corticosteroids. The patient was started on infliximab, but this was discontinued to an allergic reaction. He was then switched to etanercept. After 3 months of therapy, his swollen tender joint count decreased from 28 to $<5$. His HIV RNA peaked at 115 copies/mL while on therapy, his absolute CD4 T-cell count nadir was 236 cells $/ \mathrm{mm}^{3}$. He had no adverse events with ongoing etanercept therapy. ${ }^{51}$ In 2007, Cepeda et al ${ }^{52}$ described two HIV-infected patients with RA treated with etanercept, both of whom had very low HIV RNA levels and CD4 T-cell counts $>600$ cells/ $\mathrm{mm}^{3}$. Both patients had been treated for at least 1 year at the time of the report, and the authors observed excellent clinical responses with no complications from etanercept therapy. ${ }^{52}$ Despite these positive experiences, De Nardo et $\mathrm{al}^{53}$ reported a patient diagnosed with seronegative RA who experienced septic shock and multiorgan failure shortly after initiating etanercept and receiving the influenza vaccination.
While information about the use of TNF- $\alpha$ inhibitors in HIV-infected patients with RA is limited, this subclass of biologic agents have been used to treat other rheumatologic diseases in HIV-infected patients. Multiple case reports suggest that TNF- $\alpha$ inhibitors can be safely and effectively used in HIV patients with other rheumatologic conditions: etanercept in psoriatic arthritis, ${ }^{54}$ infliximab for psoriatic arthritis, ${ }^{55,56}$ adalimumab for psoriatic arthritis, ${ }^{57}$ infliximab for reactive arthritis, ${ }^{58,59}$ and etanercept and adalimumab for HIV-related arthritis. ${ }^{60}$ Again, despite these positive experiences, Aboulafia et $\mathrm{l}^{61}$ reported a patient with HIV infection and psoriatic arthritis (with a CD4 count of 20 cell $/ \mathrm{mm}^{3}$ and HIV load of 14,000 copies/mL) who failed multiple other less immunosuppressive therapies and responded clinically to etanercept but experienced recurrent polymicrobial infections and later died.

Beyond the use of TNF- $\alpha$ inhibitors in the treatment of HIV-infected patients with RA, very little information exists to inform us the possible safety and efficacy of other biologic agents. During our literature search, no articles were found reporting the use of rituximab, tocilizumab, or abatacept in the treatment of HIV-infected patients with RA. It has been reported that the JAK-STAT inflammatory pathway favors HIV replication, and while no studies report the clinical application of the JAK inhibitor tofacitinib in the treatment of RA, tofacitinib may promote an anti-inflammatory effect that hinders HIV virus replication. ${ }^{62}$ Given the limited information about the use of non-TNF- $\alpha$ biologic agents in the treatment of RA in HIV-infected patients, therapeutic application should proceed very cautiously.

\section{Assessing rheumatoid arthritis disease activity}

With the advent of the biologic agents and insight gathered from numerous clinical trials over the past 2 to 3 decades, the goal of RA therapy is to treat to a low disease activity state or remission. To achieve this goal, the ability to measure RA disease activity has become vital. Numerous outcomes exist to measure RA disease activity to include but not limited to changes in the erythrocyte sedimentation rate (ESR) or C-reactive protein (CRP), patient reported outcomes, and radiographic progression. How these RA disease activity measures fare when patients are chronically infected with HIV is poorly understood. The only study that we identified during our search of the literature regarding the effect of HIV infection on RA disease activity was a retrospective study of $>1,700$ South Africans diagnosed with RA, of whom 85 (4.9\%) had HIV infection subsequent to their 
diagnosis of RA. ${ }^{63}$ The conclusion reached by this study was that HIV infection leads to improvement in RA disease activity in most cases, affording most HIV-infected patients the opportunity to stop MTX. ${ }^{63}$ No significant increase in RA disease activity was recorded in six patients who commenced HAART. While the HIV-infected group with RA experienced a greater improvement in their average patient global assessment $(P=0.013)$, they also had a higher average ESR $(P<0.0001) .{ }^{63}$ As a consequence, despite improvement in RA disease activity when measured by Disease Activity Score 28 (DAS28)-CRP or DAS28-ESR, the DAS28-ESR overestimated RA disease activity by $>30 \%$ as compared to the DAS-CRP in the HIV-infected group. ${ }^{63}$ The recommendations by the authors regarding the use of the DAS28-ESR in HIV-infected patients with RA are that it is not a reliable measure of disease activity. Recognizing limitations of this study which include the retrospective design, a higher femaleto-male ratio in the HIV-infected group (9:1, as compared to a previously reported ratio of 5:1), and a prevalence of HIV infection which was 50\% lower than the estimated general population $(10.3 \%),{ }^{63}$ this study nonetheless provides preliminary insight regarding the effect HIV infection has on RA disease activity measures. Future studies could focus on how RA disease activity measures that do not rely on the ESR or CRP perform in an HIV-infected cohort.

\section{Chronic inflammation related to HIV infection}

With the implementation of HAART in the mid-1990s, management of HIV infection has transitioned from preventing and treating AIDS-related conditions to learning how to recognize and assess comorbidities that arise as a consequence of chronic inflammation related to HIV infection. New longevity because of the sustainability of remission of HIV viral replication has led to the emergence of comorbidities as a consequence of persistent immune activation. ${ }^{64}$ An aging HIV-infected population has also emerged, with $20 \%$ of the 1 million plus HIV-infected patients in the USA being older than 55 years. ${ }^{64}$ Even with long term, effective HAART, persistent low-grade inflammation, and immune activation occurs.$^{65}$ For example, soluble CD14 (sCD14) levels, a marker of monocyte activation, are typically higher in HIV-infected patients as compared with those without the virus. ${ }^{66}$ The HIV-infected adults also have a $50 \%-100 \%$ increased concentration of IL-6 than uninfected individuals. Finally, soluble CD163 levels, a marker of macrophage activation, are associated with higher risk of CAD.${ }^{66}$ Currently, HIV-infected patients experience chronic immune dysfunction, activation, and inflammation that contribute to excess morbidity and mortality. ${ }^{64}$ Since the start of this century, rheumatologists have also witnessed a similar transformation in the ongoing management of RA. With the advent of biologic agents, use of combination therapy, and changes in hygiene, previously encountered severe extraarticular disease manifestations such as vasculitis ${ }^{67}$ occur less frequently. Comorbidities related to a chronic inflammatory state, such as accelerated heart disease ${ }^{68}$ and osteoporosis, ${ }^{69}$ have become commonplace in the ongoing management of patients with RA. Immunologic abnormalities typical of the chronic inflammation associated with HIV infection have also been observed in patients with RA, such as increase IL-6 and reduced T-cell repertoire/proliferative potential. ${ }^{64}$ Rheumatologists providing care for HIV-infected patients with RA should appreciate that both conditions overlap in the immunologic dysfunction that drives inflammation and the comorbidities that might be encountered during the care of these patients. Indeed, based on the rapid evolution of knowledge in this area, it is the opinion of the authors that identifying, monitoring, and actively trying to minimize the consequences of chronic inflammation will require ongoing vigilance on the part of every clinician that cares for patients with HIV infection and RA.

Several factors likely contribute to the chronic immune activation identified in HIV-infected patients. First, although HAART is effective at suppressing most HIV replication, residual HIV replication nonetheless occurs. ${ }^{65,66}$ Although very sensitive methods are able to amplify HIV RNA from most HAART-treated HIV-infected patients, it is unclear whether this finding reflects meaningful HIV replication or passive expression of HIV from infected cells. ${ }^{70}$ Trials that have intensified HAART routinely fail to demonstrate a consistent benefit on immune activation. Thus, ongoing HIV replication may not be the sole source driving persistent immune activation in HIV-infected patients. Second, coinfection with other chronic viral infections such as with the hepatitis $\mathrm{B}$ virus, hepatitis $\mathrm{C}$ virus, and cytomegalovirus (CMV) afflict the majority of $\mathrm{HIV}$-infected patients. ${ }^{70}$ Coinfection with CMV leads to lifelong antigenic stimulation and the expansion of well-differentiated, apoptosis-resistant senescent T-cells with limited ability to replicate. ${ }^{71}$ In the setting of CMV-directed T-cell responses, it has been observed that HIV-infected individuals exhibit greater atherosclerotic burden. Treatment directed at CMV in HIV-infected patients treated with HAART led to a decline in CD8 T-cell activation at 8 weeks of therapy and persisted several weeks after cessation of therapy. ${ }^{70}$ Third, within the first weeks of 
infection, HIV leads to a significant depletion of CD4 T-cells in gut-associated lymphoid tissue and triggers gut epithelial cell apoptosis, which results in barrier dysfunction. ${ }^{70}$ Microbial translocation can occur as a result of this weakened gut barrier, and while it diminishes with HAART, it does not normalize and is associated with ongoing systemic monocyte and T-cell activation along with a poor CD4 T-cell recovery. ${ }^{70}$ Greater concentration of integrase inhibitors in the gut enlightens us on the significance of microbial translocation as a recent trial demonstrated improvement in sCD14 and high-sensitive CRP levels independent of HIV suppression or immune recovery. ${ }^{65}$ Finally, lifestyle factors such as being sedentary, smoking, or intravenous drug use appear to independently contribute to ongoing chronic immune activation and inflammation. ${ }^{65,66}$

The consequences of chronic immune activation and inflammation in HIV infection are akin to those observed in patients with RA. There is a heightened risk of atherosclerotic coronary artery disease. ${ }^{65,66}$ As in RA, HIV infection independently imparts at least a $50 \%$ increase in the relative risk of myocardial infarction. ${ }^{64,66,72}$ In those with sustained control of HIV replication with HAART, the inflammatory burden of HIV infection has an effect similar to traditional risk factors, such as hypertension and diabetes. ${ }^{72}$ There is accelerated bone loss in HIV-infected patients. ${ }^{65,66}$ Lower bone density is more prevalent in HIV-infected patients ${ }^{64}$ and higher rates of osteoporosis are observed when compared to the general population. ${ }^{73}$ HAART has been associated with a decrease in bone mineral density, mainly in the first year of therapy, but it is unclear whether this translates into an increased risk of fracture. ${ }^{73} \mathrm{HIV}$-infected patients are also at increased risk of anemia, ${ }^{65}$ osteonecrosis, ${ }^{73}$ chronic kidney disease, liver disease, and neurocognitive deficits/decline. ${ }^{66,70}$

What can be done to mitigate the comorbidities described earlier in HIV-infected patients with RA? Though our knowledge in this area continues to grow, some empiric recommendations can be made. First, promote lifestyle changes. Avoiding a sedentary lifestyle and exercising reduces inflammation and have benefits beyond a reduction observed in biomarkers. ${ }^{65}$ Tobacco cessation is also very likely beneficial, again as observed in other illnesses associated with chronic inflammation. Second, avoid medications with adverse effects on the various comorbidities discussed earlier. When treating HIV infection, abacavir-containing regimens appear to have a smaller impact on endothelial activation markers and vascular inflammation as compared to regimens without this medication. ${ }^{74}$ Low vitamin $\mathrm{D}$ levels are associated with use of efavirenz. ${ }^{73}$ HIV treatment guidelines are now integrating such findings in their recommendations, as medication regimens need to balance long-term toxicity with antiviral potency. ${ }^{66}$ Third, more aggressive treatment of the various comorbidities observed may be effective. An observational study demonstrated that HAART with rosuvastatin was associated with significantly greater decrease in high-sensitive CRP and TNF- $\alpha$ than HAART alone. ${ }^{75}$ Several trials have demonstrated the safety and efficacy of bisphosphonates in improving bone mineral density in HIV-infected patients. ${ }^{73}$ Finally, initiation of HAART earlier in the disease course may mitigate the burden of chronic inflammation. ${ }^{70}$ It has been observed that soluble markers of monocyte activation remain persistently elevated early in HAART among those with chronic HIV infection; however, these markers appear to return to near normal levels in those with acute HIV infection. ${ }^{70}$ Lower T-cell activation has also been observed in those who receive HAART within 6 months of confirmed seroconversion as compared to those started 3 years later, although all HIV-infected patients had higher T-cell activation compared to uninfected patients. ${ }^{70}$ While these data are intriguing, it is important to consider that earlier initiation of HAART could significantly increase the cost of treating HIV infection and may place the patient at increased risk of toxicity.

\section{The immune reconstitution inflammatory syndrome}

The IRIS has been described as the paradoxical worsening of a pre-existing infectious process after initiation of HAART. ${ }^{64}$ The pre-existing infection may be one that has been diagnosed and treated or was subclinical and unmasked as the host's immunity regained ability to mount an inflammatory response. ${ }^{64}$ It is a topic of great interest and when it occurs creates a challenge in treating HIV infection. Risk factors for the development of IRIS are male sex, younger age, lower CD4 T-cell count and percentage at the start of HAART, higher HIV RNA at the start of HAART, more rapid initial fall in HIV RNA on HAART, antiretroviral naivety at the time an OI is diagnosed, and a shorter interval between the start of therapy for OI and HAART. ${ }^{76}$ IRIS can also lead to the exacerbation of a previously occult or quiescent autoinflammatory disease, such as RA. Multiple cases can now be found in the literature $^{27,77-79}$ describing the onset of RA after initiation of HAART as the immune system reconstitutes. Among larger cohorts of HIV-infected patients, ${ }^{8,79,80}$ RA may develop after HAART is initiated but in a very small percentage. In a Taiwanese cohort of $>3,600$ patients, five patients $(0.14 \%)$ developed RA after HAART was initiated.$^{80}$ The average time 
to the onset of arthritic symptoms was 67 months. ${ }^{80}$ Overall, the emergence or worsening of RA as a consequence of IRIS appears to be a very infrequent occurrence. ${ }^{81}$ Although unlikely to be encountered in clinical practice, HIV-infected patients with RA who experience worsening of their RA as a consequence of IRIS will likely require cautious advancement of either DMARDs or biologic response modifiers. Should this occur, there is very little information to guide which pharmacologic options best balance immunosuppression with risk of infection in an already immunocompromised host.

\section{Conclusion}

Within the past 2 to 3 decades, HIV has been transformed from a once uniformly fatal disease to now a manageable but complex multisystem illness. In the pre-HAART era, reports suggested that HIV-infected patients with RA would experience remission of their disease or would not develop this inflammatory arthritis. In the past 2 decades, it has now become clear that RA can be developed in HIV-infected patients at any time, independent of therapy with HAART, although this appears to be a rare occurrence. Choosing the right medication to treat symptoms related to RA but avoiding excess weakening of the immune system remains a significant clinical challenge. It appears that immunomodulating agents such as HCQ and SSZ might best balance safety with efficacy, making them reasonable first choices for therapy in HIV-infected patients with RA. Corticosteroids such as prednisone may also be reasonable first-line therapies but could increase the risk of osteonecrosis, a complication more likely to be observed in HIV-infected patients independent of corticosteroid use. More immune suppressing agents such as MTX, LFN, and CsA may also balance safety with efficacy, but data are limited. Among all the biologic response modifiers, TNF- $\alpha$ inhibitors may be a safe and effective option for treatment of RA in patients with HIV, as long as HIV infection is well controlled with ongoing HAART therapy. There are no available data regarding the use of non-TNF- $\alpha$ biologic agents in the treatment of RA in the setting of HIV. The monitoring of RA disease activity is challenging as only one retrospective study has been published in this area, which suggests that ESR values may lead to the overestimation of disease activity. Those with HIV infection and RA can experience comorbidities such as accelerated heart disease and osteoporosis, comorbidities that can occur in either illness alone. These comorbidities arise as a consequence of the chronic inflammatory state that each illness generates, and while limited information exists on how to best minimize the clinical effects of chronic inflammation, interventions such as smoking cessation, exercising more, and pursuing good health may be beneficial. A concern of practitioners managing HIV-infected patients who start HAART would be the development of IRIS, but it appears that IRIS has a small influence on triggering the onset or worsening of RA. Going forward, with the current limitations in our knowledge on how to manage RA in those with HIV infection, studies beyond case reports and case series are desperately needed. Retrospective studies to address these limitations would serve as a good start, and though they are limited by various forms of bias and confounding, the rarity in which patients will have both RA and HIV infection would potentially make prospective studies difficult due to the larger numbers of patients needed for enrollment.

\section{Acknowledgment}

The views expressed in this material are those of the authors and do not reflect the official policy or position of the US government, the Department of Defense, or the Department of the Air Force.

\section{Disclosure}

The authors report no conflicts of interest in this work.

\section{References}

1. Shah D, Flanigan T, Lally E. Routine screening for HIV in rheumatology practice. J Clin Rheumatol. 2011;17(3):154-156.

2. Yao Q, Frank M, Glynn M, Altman RD. Rheumatic manifestations in HIV-1 infected in-patients and literature review. Clin Exp Rheumatol. 2008;26(5):799-806.

3. Lawson $\mathrm{E}$, Walker-Bone $\mathrm{K}$. The changing spectrum of rheumatic disease in HIV infection. Br Med Bull. 2012;103(1):203-221.

4. Bijlsma JS, Derksen RW, Huber-Bruning O, Borleffs JC. Does AIDS 'cure' rheumatoid arthritis. Ann Rheum Dis. 1988;47:350-351.

5. Reveille JD, Williams FM. Infection and musculoskeletal conditions: rheumatologic complications of HIV infection. Best Pract Res Clin Rheumatol. 2006;20(6):1159-1179.

6. Black A, Madhok R. The coexistence of classic RA and AIDS. J Rheumatol. 1992;19(9):1484-1485.

7. Ornstein MH, Kerr LD, Spiera H. A reexamination of the relationship between active rheumatoid arthritis and the acquired immunodeficiency syndrome. Arthritis Rheum. 1995;38(11):1701-1706.

8. Iordache L, Launay $\mathrm{O}$, Bouchaud $\mathrm{O}$, et al. Autoimmune diseases in HIV-infected patients: 52 cases and literature review. Autoimmun Rev. 2014;13(8):850-857.

9. Zandman-Goddard G, Shoenfeld Y. HIV and autoimmunity. Autoimmun Rev. 2002;1(6):329-337.

10. du Toit R, Whitelaw D, Taljaard JJ, du Plessis L, Esser M. Lack of specificity of anticyclic citrullinated peptide antibodies in advanced human immunodeficiency virus infection. J Rheumatol. 2011;38(6):1055-1060.

11. Romic Z, Derek L, Burek V, et al. Anti-cyclic citrullinated peptide and rheumatoid factor in HIV positive patients. Rheumatol Int 2011;31(12):1607-1610.

12. Saag KG, Teng GG, Patkar NM, et al; American College of Rheumatology. American College of Rheumatology 2008 recommendations for the use of nonbiologic and biologic disease-modifying antirheumatic drugs in rheumatoid arthritis. Arthritis Rheum. 2008;59(6):762-784. 
13. Singh JA, Furst DE, Bharat A, et al. 2012 update of the 2008 American College of Rheumatology recommendations for the use of diseasemodifying antirheumatic drugs and biologic agents in the treatment of rheumatoid arthritis. Arthritis Care Res. 2012;64(5):625-639.

14. Chiang G, Sassaroli M, Louie M, Chen H, Stecher VJ, Sperber K. Inhibition of HIV-1 replication by hydroxychloroquine. Clin Ther. 1996;18(6):1080-1092.

15. Sperber K, Kalb TH, Stecher VJ, Banerjee R, Mayer L. Inhibition of human immunodeficiency virus type 1 replication by hydroxychloroquine in T cells and monocytes. AIDS Res Hum Retroviruses. 1993;9(1):91-98.

16. Savarino A, Gennero L, Chen HC, et al. Anti-HIV effects of chloroquine. AIDS. 2001;15(17):2221-2229.

17. Tsai WP, Nara PL, Kung HF, Oroszlan S. Inhibition of human immunodeficiency virus infectivity by chloroquine. AIDS Res Hum Retroviruses. 1990;6(4):481-489.

18. Ornstein $\mathrm{MH}$, Sperber K. The antiinflammatory and antiviral effects of hydroxychloroquine in two patients with acquired immunodeficiency syndrome and active inflammatory arthritis. Arthritis Rheum. 1996;39:157-161.

19. Paton NI, Goodall RL, Dunn DT, et al. Effects of hydroxychloroquine on immune activation and disease progression among HIV-infected patients not receiving antiretroviral therapy: a randomized controlled trial. JAMA. 2012;308(4):353-361.

20. Sperber K, Louie M, Kraus T, et al. Hydroxychloroquine treatment of patients with human immunodeficiency virus type 1. Clin Ther. 1995;17(4):622-636.

21. Piconi S, Parisotto S, Rizzardini G, et al. Hydroxychloroquine drastically reduces immune activation in HIV- infected, antiretroviral therapy-treated, immunological nonresponders. Blood. 2011;118(12):3263-3272.

22. Sperber K, Quraishi H, Kalb TH, Panja A, Stecher V, Mayer L. Selective regulation of cytokine secretion by hydroxychloroquine: inhibition of interleukin 1 alpha (IL-1-alpha) and IL-6 in human monocytes and T cells. J Rheumatol. 1993;20(5):803-808.

23. Gilman AL, Beams F, Tefft M, Mazumder A. The effect of hydroxychloroquine on alloreactivity and its potential use for graft-versus-host disease. Bone Marrow Transplant. 1996;17(6):1069-1075.

24. Khalili H, Dastan F, Dehghan Manshadi SA. A case report of hearing loss post use of hydroxychloroquine in a HIV-infected patient. Daru. 2014;22(1):20.

25. Bortoli R, Santiago M. Chloroquine ototoxicity. Clin Rheumatol. 2007;26(11):1809-1810.

26. Riva DA, Fernández-Larrosa PN, Dolcini GL, Martínez-Peralta LA, Coulombié FC, Mersich SE. Two immunomodulators, curcumin and sulfasalazine, enhance IDV antiretroviral activity in HIV-1 persistently infected cells. Arch Virol. 2008;153(3):561-565.

27. Siva C, Brasington RD. Worsening of arthritis with antiretroviral therapy: the coexistence of rheumatoid arthritis and human immunodeficiency virus infection revisited. J Clin Rheumatol. 2001;7(1):42-46.

28. Njobvu PD, McGill PE. Sulphasalazine in the treatment of HIV-related spondyloarthropathy. Br J Rheumatol. 1997;36(3):403-404.

29. Disla E, Rhim HR, Reddy A, Taranta A. Improvement in CD4 lymphocyte count in HIV-Reiter's syndrome after treatment with sulfasalazine. J Rheumatol. 1994;21(4):662-664.

30. National Institute of Allergy and Infectious Diseases (NIAID); United States: Federal Government [webpage on the Internet]. A Phase I Study of Methotrexate for HIV Infection. Bethesda, MD:National Library of Medicine (US). [cited August 15, 1999]. Available from: https://clinicaltrials.gov/ct2/show/NCT00000834. Accessed August 15, 2015.

31. Actgnetwork.org [homepage on the Internet]. US Department of Health and Human Services, the National Institutes of Health, the National Institute of Allergy and Infectious Diseases, and the Division of AIDS. [cited August 15, 2015]. Available from: https://actgnetwork.org/study/ a5314-mtx-hiv-positives. Accessed August 15, 2015.
32. Maurer TA, Zackheim HS, Tuffanelli L, Berger TG. The use of methotrexate for treatment of psoriasis in patients with HIV infection. J Am Acad Dermatol. 1994;31(2 pt 2):372-375.

33. Read SW, DeGrezia M, Ciccone EJ, et al. The effect of leflunomide on cycling and activation of T-cells in HIV-1-infected participants. PLoS One. 2010;5:e11937.

34. Schlapfer E, Fischer M, Ott P, Speck RF. Anti-HIV-1 activity of leflunomide: a comparison with mycophenolic acid and hydroxyurea. AIDS. 2003;17:1613-1620.

35. Chen S, Zhao X, Tan J, et al. Structure-based identification of small molecule compounds targeting cell cyclophilin A with anti-HIV-1 activity. Eur J Pharmacol. 2007;565:54-59.

36. Mlynar E, Bevec D, Billich A, Rosenwirth B, Steinkasserer A. The nonimmunosuppressive cyclosporin A analogue SDZ NIM 811 inhibits cyclophilin A incorporation into virions and virus replication in human immunodeficiency virus type 1-infected primary and growth-arrested T cells. J Gen Virol. 1997;78:825-835.

37. Ptak RG, Gallay PA, Jochmans D, et al. Inhibition of human immunodeficiency virus type 1 replication in human cells by Debio-025, a novel cyclophilin binding agent. Antimicrob Agents Chemother. 2008;52:1302-1317.

38. Levy R, Jais JP, Tourani JM, Even P, Andrieu JM. Long-term follow-up of HIV positive asymptomatic patients having received cyclosporin A. Adv Exp Med Biol. 1995;374:229-234.

39. Calabrese LH, Lederman MM, Spritzler J, et al; AIDS Clinical Trials Group 334 Investigators. Placebo-controlled trial of cyclosporin-A in HIV-1 disease: implications for solid organ transplantation. J Acquir Immune Defic Syndr. 2002;29:356-362.

40. Saulsbury FT, Bringelsen KA, Normansell DE. Effects of prednisone on human immunodeficiency virus infection. South Med J. 1991;84(4):431-435.

41. McComsey GA, Whalen CC, Mawhorter SD, et al. Placebocontrolled trial of prednisone in advanced HIV-1 infection. AIDS. 2001;15(3):321-327.

42. Wallis RS, Kalayjian R, Jacobson JM, et al. A study of the immunology, virology, and safety of prednisone in HIV-1-infected subjects with CD4 cell counts of 200 to $700 \mathrm{~mm}(-3)$. J Acquir Immune Defic Syndr. 2003;32(3):281-286.

43. Kasang C, Ulmer A, Donhauser N, et al. HIV patients treated with low-dose prednisolone exhibit lower immune activation than untreated patients. BMC Infect Dis. 2012;12:14

44. Scribner AN, Troia-Cancio PV, Cox BA, et al. Osteonecrosis in HIV: a case-control study. J Acquir Immune Defic Syndr. 2000;25: 19-25.

45. Glesby MJ, Hoover DR, Vaamonde CM. Osteonecrosis in patients infected with human immunodeficiency virus: a case-control study. J Infect Dis. 2001;184:519-523.

46. Lexi-Comp Inc. Lexicomp Online, Interactions. Hudson, OH: LexiComp Inc.; 2015.

47. Hall JJ, Hughes CA, Foisy MM, Houston S, Shafran S. Iatrogenic Cushing syndrome after intra-articular triamcinolone in a patient receiving ritonavir-boosted darunavir. Int J STD AIDS. 2013;24(9): $748-752$.

48. Johnson SR, Marion AA, Vrchoticky T, Emmanuel PJ, LujanZilbermann J. Cushing syndrome with secondary adrenal insufficiency from concomitant therapy with ritonavir and fluticasone. $J$ Pediatr. 2006;148(3):386-388.

49. Izzedine H, Launay-Vacher V, Baumelou A, Deray G. Antiretroviral and immunosuppressive drug-drug interactions: an update. Kidney Int. 2004;66(2):532-541.

50. American College of Rheumatology [webpage on the Internet]. 2015 American College of Rheumatology Guideline for the Treatment of Rheumatoid Arthritis. [cited August 15, 2015]. Available from: http:// www.rheumatology.org/Practice-Quality/Clinical-Support/ClinicalPractice-Guidelines/Rheumatoid-Arthritis. Accessed August 15, 2015 . 
51. Kaur PP, Chan VC, Berney SN. Successful etanercept use in an HIV-positive patient with rheumatoid arthritis. J Clin Rheumatol. 2007;13(2):79-80.

52. Cepeda EJ, Williams FM, Ishimori ML, Weisman MH, Reveille JD. The use of anti-tumour necrosis factor therapy in HIV-positive individuals with rheumatic disease. Ann Rheum Dis. 2008;67(5): 710-712.

53. De Nardo P, Bellagamba R, Corpolongo A, et al. Septic shock after seasonal influenza vaccination in an HIV-infected patient during treatment with etanercept for rheumatoid arthritis: a case report. Clin Vaccine Immunol. 2013;20(5):761-764.

54. Linardaki G, Katsarou O, Ioannidou P, Karafoulidou A, Boki K. Effective etanercept treatment for psoriatic arthritis complicating concomitant human immunodeficiency virus and hepatitis $\mathrm{C}$ virus infection. J Rheumatol. 2007;34(6):1353-1355.

55. Bartke U, Venten I, Kreuter A, Gubbay S, Altmeyer P, Brockmeyer NH. Human immunodeficiency virus-associated psoriasis and psoriatic arthritis treated with infliximab. Br J Dermatol. 2004;150(4):784-786. Erratum in: Br J Dermatol. 2004 Jun;150(6):1235. Gabbay, S corrected to Gubbay, S.

56. Sellam J, Bouvard B, Masson C, et al. Use of infliximab to treat psoriatic arthritis in HIV-positive patients. Joint Bone Spine. 2007;74(2):197-200

57. Lindsey SF, Weiss J, Lee ES, Romanelli P. Treatment of severe psoriasis and psoriatic arthritis with adalimumab in an HIV-positive patient J Drugs Dermatol. 2014;13(7):869-871.

58. Gaylis N. Infliximab in the treatment of an HIV positive patient with Reiter's syndrome. J Rheumatol. 2003;30(2):407-411.

59. Gaylis NB. Long-term follow-up of an HIV-infected patient with reactive arthritis treated with infliximab. $J$ Clin Rheumatol. 2012;18(3):153-154.

60. Almoallim H, Jali I, Wali G. Successful use of antitumor necrosis factor-alpha biological therapy in managing human immunodeficiency virus-associated arthritis: three case studies from Saudi Arabia. Joint Bone Spine. 2013;80(4):426-428.

61. Aboulafia DM, Bundow D, Wilske K, Ochs UI. Etanercept for the treatment of human immunodeficiency virus-associated psoriatic arthritis. Mayo Clin Proc. 2000;75(10):1093-1098.

62. Gavegnano C, Detorio M, Montero C, Bosque A, Planelles V, Schinazi RF. Ruxolitinib and tofacitinib are potent and selective inhibitors of HIV-1 replication and virus reactivation in vitro. Antimicrob Agents Chemother. 2014;58(4):1977-1986.

63. Tarr G, Makda M, Musenge E, Tikly M. Effect of human immunodeficiency virus infection on disease activity in rheumatoid arthritis: a retrospective study in South Africans. J Rheumatol. 2014;41(8):1645-1649.

64. Calabrese LH, Kirchner E. HIV Infection: What Rheumatologists Need to Know. Atlanta: The Rheumatologist; 2015.

65. Erlandson KM, Campbell TB. Inflammation in chronic HIV infection: what can we do? J Infect Dis. 2015;212(3):339-342.
66. Deeks SG, Lewin SR, Havlir DV. The end of AIDS: HIV infection as a chronic disease. Lancet. 2013;382(9903):1525-1533.

67. Bartels CM, Bell CL, Shinki K, Rosenthal A, Bridges AJ. Changing trends in serious extra-articular manifestations of rheumatoid arthritis among United States veterans over 20 years. Rheumatology (Oxford). 2010;49(9):1670-1675.

68. Crowson CS, Liao KP, Davis JM 3rd, et al. Rheumatoid arthritis and cardiovascular disease. Am Heart J. 2013;166(4):622.e-628.e.

69. Bultink IE, Vis M, van der Horst-Bruinsma IE, Lems WF. Inflammatory rheumatic disorders and bone. Curr Rheumatol Rep. 2012;14(3) 224-230.

70. Hunt PW. HIV and inflammation: mechanisms and consequences. Curr HIV/AIDS Rep. 2012;9(2):139-147.

71. Deeks SG. HIV infection, inflammation, immunosenescence, and aging. Annu Rev Med. 2011;62:141-155.

72. Freiberg MS, Chang CC, Kuller LH, et al. HIV infection and the risk of acute myocardial infarction. JAMA Intern Med. 2013;173(8): 614-622.

73. Gedmintas L, Solomon DH. HIV and its effects on bone: a primer for rheumatologists. Curr Opin Rheumatol. 2012;24(5):567-575.

74. Hileman CO, Wohl DA, Tisch DJ, Debanne SM, McComsey GA. Short communication: initiation of an abacavir-containing regimen in HIVinfected adults is associated with a smaller decrease in inflammation and endothelial activation markers compared to non-abacavir-containing regimens. AIDS Res Hum Retroviruses. 2012;28(12):1561-1564.

75. Calza L, Vanino E, Salvadori C, et al. Tenofovir/emtricitabine/efavirenz plus rosuvastatin decrease serum levels of inflammatory markers more than antiretroviral drugs alone in antiretroviral therapy-naive HIVinfected patients. HIV Clin Trials. 2014;15(1):1-13.

76. Murdoch DM, Venter WD, Van Rie A, Feldman C. Immune reconstitution inflammatory syndrome (IRIS): review of common infectious manifestations and treatment options. AIDS Res Ther. 2007;4:9.

77. Bell C, Nelson M, Kaye S. A case of immune reconstitution rheumatoid arthritis. Int J STD AIDS. 2002;13(8):580-581.

78. McLeod DS, Woods ML, Kandiah DA. Immune reconstitution inflammatory syndrome manifesting as development of multiple autoimmune disorders and skin cancer progression. Intern Med J. 2011;41(9):699-703.

79. Calabrese LH, Kirchner E, Shrestha R. Rheumatic complications of human immunodeficiency virus infection in the era of highly active antiretroviral therapy: emergence of a new syndrome of immune reconstitution and changing patterns of disease. Semin Arthritis Rheum. 2005;35:166-174.

80. Yang JJ, Tsai MS, Sun HY, et al. Autoimmune diseases-related arthritis in HIV-infected patients in the era of highly active antiretroviral therapy. J Microbiol Immunol Infect. 2015;48(2):130-136.

81. Cunha BM, Mota LM, Pileggi GS, Safe IP, Lacerda MV. HIV/AIDS and rheumatoid arthritis. Autoimmun Rev. 2015;14(5):396-400.

\section{Publish your work in this journal}

Open Access Rheumatology Research and Reviews is an international, peer-reviewed, open access journal, publishing all aspects of clinical and experimental rheumatology in the clinic and laboratory including the following topics: Pathology, pathophysiology of rheumatological diseases; Investigation, treatment and management of rheumatological

\section{Dovepress}

diseases; Clinical trials and novel pharmacological approaches for the treatment of rheumatological disorders. The manuscript management system is completely online and includes a very quick and fair peerreview system, which is all easy to use. Visit http://www.dovepress.com/ testimonials.php to read real quotes from published authors. 